\title{
The Role of Morphine in Animal Models of Human Cancer: Does Morphine Promote or Inhibit the Tumor Growth?
}

\author{
Sabrina Bimonte, ${ }^{1}$ Antonio Barbieri, ${ }^{1}$ Giuseppe Palma, ${ }^{1,2}$ and Claudio Arra ${ }^{1}$ \\ ${ }^{1}$ Istituto Nazionale per lo Studio e la Cura dei Tumori, "Fondazione G. Pascale," IRCCS, Via Mariano Semmola, 80131 Naples, Italy \\ ${ }^{2}$ Istituto di Endocrinologia e Oncologia Sperimentale del Consiglio Nazionale delle Ricerche, Dipartimento di Biologia e Patologia \\ Cellulare e Molecolare "L. Califano," Università degli Studi di Napoli "Federico II," Via Pansini 5, 80131 Napoli, Italy
}

Correspondence should be addressed to Sabrina Bimonte; s.bimonte@istitutotumori.na.it

Received 16 July 2013; Accepted 29 July 2013

Academic Editor: Monica Fedele

Copyright (C) 2013 Sabrina Bimonte et al. This is an open access article distributed under the Creative Commons Attribution License, which permits unrestricted use, distribution, and reproduction in any medium, provided the original work is properly cited.

\begin{abstract}
Morphine, a highly potent analgesic agent, is widely used to relieve pain and suffering of patients with cancer. Additionally, it has been reported that morphine is important in the regulation of cancerous tissue. Morphine relieves pain by acting directly on the central nervous system, although its activities on peripheral tissues are responsible for many adverse side effects. For these reasons, it is very important also to understand the role of morphine in cancer treatment. The published literature reporting the effect of morphine on tumor growth presents some discrepancies, with reports suggesting that morphine may either promote or inhibit the tumor growth. It has been also demonstrated that morphine modulates angiogenesis which is important for primary tumour growth, invasiveness, and the development of metastasis. This review will focus on the latest findings on the role of morphine in the regulation of cancer cell growth and angiogenesis.
\end{abstract}

\section{Introduction}

Morphine is used to relieve pains of patients with cancer in terminal phases, in order to improve quality of life [1]. Morphine is an opiate-based drug isolated for the first time in 1803 by Friedrich W. Sertürner [2]. It has been shown that morphine explains its function by acting through opioid receptors, $\mu, \delta$, and $\kappa$, which are localized in the brain $[3,4]$. Morphine relieves pain by acting directly on central nervous system (CNS), although its activity on peripheral tissue leads to many secondary complications, including addiction, respiratory depression, and tolerance. Apart from these severe effects, morphine is still considered the most effective drug clinically available for the management of severe pain associated with cancer [5]. Several experimental studies performed on cancer cell lines and mouse models showed that morphine can also play a role in the regulation of cancer cell growth. Unfortunately, the results obtained by these studies are still contradictory. Some reports demonstrated that morphine inhibited the growth of various human cancer cell lines [6-12] or animal models [13-16]. On the contrary, other studies proved that morphine increased tumor cell growth in in vivo
$[17,18]$ or in vitro [19] models. According to some studies, morphine at clinically relevant doses stimulated angiogenesis in vitro [20] and tumour growth in breast cancer mouse model [21]. It has been demonstrated that morphine modulates angiogenesis which is important for primary tumour growth, invasiveness, and the development of metastasis. For these reasons, there is a dilemma about the effects of morphine on cancer cell growth and angiogenesis.

This review will focus on the latest findings on the role of morphine in the regulation of cancer cell growth and angiogenesis.

\section{Morphine Affects Tumor Growth and Apoptosis}

The role of morphine in the regulation of tumor cell growth is not yet correctly established. Several xenograft mouse models were generated to study cancer cell growth-promoting or inhibiting effects of morphine. Tegeder et al. [13] generated mouse models of breast cancer by subcutaneous injection of MCF-7 and MDA-MB231 cells in NMRI-nu/nu mice. In these 
mice, morphine, intraperitoneally injected, significantly reduced tumor growth through a p53-dependent mechanism. Additionally, in these mice, naloxone, an opioid inverse agonist, increased the growth-inhibitory effects of morphine. Similar results were obtained in rat model of colon cancer generated by intraperitoneal injection of colon cancer cells in Fisher 244 rats. In these animals, subcutaneous administration of morphine leads to significant decrease in the hepatic tumor burden. Morphine inhibited not only tumor growth but also metastasis in melanoma mouse model generated by subcutaneous injection of B16-BL6 cells into the hind paws of C57BL mice [15]. Another group, demonstrated that morphine inhibited tumor metastasis formation when it was administered intraperitoneally in mouse model of colon cancer [16]. On the contrary, several experimental studies demonstrated that morphine increased tumor growth. Gupta et al., in orthotropic mouse model of breast cancer obtained by injection of MCF-7 cells into the mammary fat pads of nude mice, demonstrated that morphine, in clinically relevant doses, increased tumor growth. This was associated with increased angiogenesis and inhibition of apoptosis and promotion of cell cycle progression [20]. In this study, it was also reported that naloxone itself had no significant effect on angiogenesis. Our preliminary data, obtained by in vitro and in vivo experiments using MDA.MB231 breast cancer cells, seems to validate this hypothesis (Bimonte et al., unpublished data). According to these results, in another study, it was demonstrated that morphine, subcutaneously administrated in mice, increased the tumor growth in mouse model of leukaemia and sarcoma. In these mice, morphine played also a general immunosuppressive role [22].

These contrasting results are probably associated with different concentration and/or time of administration of morphine. In fact, in vitro and in vivo studies demonstrated that tumor-enhancing effects with morphine occur after administration of low daily doses or single dose of morphine [23], while tumor suppression occurs after chronic high doses of morphine $[11,15,16]$.

It has also been demonstrated that the $\mu$-opioid receptor, by which morphine exerts its action, directly regulates tumor growth and metastasis. On the basis of these results, different mechanisms of opioid receptor-mediated influence of morphine on tumor growth have been proposed. Morphine, as mentioned above, after binding to the $\mu$-opioid receptor, regulates cell cycle progression by stimulating mitogen-activated protein kinase (MAPK)/extracellular growth factor (Erk) pathways [20]. Alternatively, morphine can mediate apoptosis by activating phosphatidylinositol 3-kinase (PI3K)/protein kinase B (Akt) pathway [24]. Additionally, morphine, by the upregulation of urokinase plasminogen activator (uPA) expression, induces metastasis formation [25], while, by transactivation of VEGF receptor, it induces angiogenesis [26]. Finally, morphine affects also the function of T lymphocytes, leading to immunosuppression [27].

It has been proposed that morphine plays also a role in tumor apoptosis. Apoptosis is a form of cell death in which a programmed sequence of events leads to the elimination of cells without releasing harmful substances into the surrounding area. It is noted that apoptosis is regulated by two pathways: the mitochondrial-mediated pathway (intrinsic) [28] and death receptor-mediated pathway (extrinsic) [29]. It is noted that in cancer cells apoptosis is deregulated, and this leads to quick proliferation and tumor growth [30, 31]. Morphine was shown to induce apoptosis of macrophages, T lymphocytes, and human endothelial cells [32, 33]. Experiments performed on human tumor cell lines demonstrated that morphine in high concentration induces apoptosis and inhibits cancer cell growth by activation of different signal pathways involving caspase 3/9, cytochrome c, and sigma-2 receptor. On the contrary, it has been demonstrated that morphine can inhibit apoptosis. Additionally in SH-SY5Y cells, morphine has antiapoptotic effect by antagonizing doxorubicin [34]. These discrepancies, also in these cases, are associated with different cell line tumor type used and/or in vivo dose/time of morphine administrated.

\section{Morphine Regulates Angiogenesis and Metastasis Formation}

Recent data demonstrated a role of morphine in angiogenesis. Angiogenesis is required for invasive tumor growth and metastasis and represents an important point in the control of cancer progression. Proangiogenic activity of morphine was demonstrated in the MCF-7 breast cancer model. In these mice, morphine at clinically relevant concentrations enhanced tumor neovascularization [20]. In an animal model of hormone-dependent breast cancer, it has also been demonstrated that morphine promoted activation of vascular endothelial growth factor (VEGF) receptor and increased metastasis [21, 27]. It has been proposed that morphine explains its proangiogenic activity by the stimulation of mitogen-activated protein kinase (MAPK) signalling pathway via G proteincoupled receptors and nitric oxide (NO). Alternatively, several in vivo studies provided evidence that morphine can induce tumor growth by the upregulation of cyclooxygenase2 (COX-2) [35-38] and/or prostaglandin E2-mediated stimulation of angiogenesis [39-42]. On the contrary, several in vivo and in vitro studies demonstrated that morphine can inhibit angiogenesis by the regulation of different pathways $[8,32,43,43-50]$. These different results can be due to different experimental conditions (cell line tumor type used and/or dose/time of morphine). Morphine plays a role not only in tumor cell growth but also in metastasis formation, which is the main process related to most cancer deaths and failure in cancer treatment $[51,52]$. The process which leads to metastasis formation initiated with migration of cancer cells through the extracellular matrix (ECM). Both pro- and antimigratory effects have been reported for morphine. Specifically, it has been shown that morphine significantly reduces the adhesion, invasion, and metastasis of metastatic colon 26-L5 carcinoma cells [16], by the regulation of matrix metalloproteinases (MMPs). On the contrary, morphine can promote invasion, metastasis formation and migration of cancer cells by the upregulation of MMPs in breast and lung cancer [52, 53]. Finally, in MCF-7 breast cancer cells $[50,54]$ and in $H 729$ cancer cells [55], morphine treatments lead to the upregulation of urokinase plasminogen activator (uPA) which promotes migration of cancer cells through the ECM. 


\section{Conclusions}

Several studies provided evidence that morphine can affect tumor growth by acting with different mechanisms, including tumor cells or endothelial cells or growth factors secreted by meditation of CNS. Unfortunately, the results obtained from both in vitro and in vivo studies are so far conflicting. Some reports suggested that morphine may promote the tumor growth by inhibiting apoptosis and by promoting angiogenesis and migration of tumor cells. On the contrary, it has been demonstrated that morphine may also exert proapoptotic and antiangiogenic effects. These different results can be associated with the different doses of morphine administrated, with different models used and different cancer. For these reasons, it is very important for the management of severe pain associated with cancer to consider accurately the dose and route of administration of morphine. Further studies will be necessary to establish if morphine is an inhibitor of tumor growth or whether it promotes cancer.

\section{Disclosure}

The authors have no other relevant affiliations or financial interests with any organization or entity. No writing assistance was used in the production of the paper.

\section{Acknowledgments}

The authors thank Massimiliano Spinelli for kind help in providing informatics assistance. This work was supported by the $5 \mathrm{x}$ mille and current research programs of the Istituto Nazionale per lo Studio e la Cura dei Tumori, "Fondazione G. Pascale," IRCCS, Naples, Italy.

\section{References}

[1] J. N. Lickiss, “Approaching cancer pain relief," European Journal of Pain, vol. 5, pp. 5-14, 2001.

[2] R. Schmitz, "Friedrich Wilhelm Sertürner and the discovery of morphine," Pharmacy in history, vol. 27, no. 2, pp. 61-74, 1985.

[3] G. W. Pasternak, "Pharmacological mechanisms of opioid analgesics," Clinical Neuropharmacology, vol. 16, no. 1, pp. 1-18, 1993.

[4] B. L. Kieffer and C. Gavériaux-Ruff, "Exploring the opioid system by gene knockout," Progress in Neurobiology, vol. 66, no. 5, pp. 285-306, 2002.

[5] P. W. Mantyh, "Cancer pain and its impact on diagnosis, survival and quality of life," Nature Reviews Neuroscience, vol. 7, no. 10, pp. 797-809, 2006.

[6] N. Sueoka, E. Sueoka, S. Okabe, and H. Fujiki, "Anti-cancer effects of morphine through inhibition of tumour necrosis factor- $\alpha$ release and mRNA expression," Carcinogenesis, vol. 17, no. 11, pp. 2337-2341, 1996.

[7] R. Maneckjee and J. D. Minna, "Opioids induce while nicotine suppresses apoptosis in human lung cancer cells," Cell Growth and Differentiation, vol. 5, no. 10, pp. 1033-1040, 1994.

[8] A. Pasi, B. Qu, R. Steiner, H.-J. Senn, W. Bar, and F. S. Messiha, "Angiogenesis: modulation with opioids," General Pharmacology, vol. 22, no. 6, pp. 1077-1079, 1991.
[9] A. Hatzoglou, E. Bakogeorgou, E. Papakonstanti, C. Stournaras, D. S. Emmanouel, and E. Castanas, "Identification and characterization of opioid and somatostatin binding sites in the opossum kidney (OK) cell line and their effect on growth," Journal of Cellular Biochemistry, vol. 63, no. 4, pp. 410-421, 1996.

[10] A. Hatzoglou, L. Ouafik, E. Bakogeorgou, K. Thermos, and E. Castanas, "Morphine cross-reacts with somatostatin receptor SSTR2 in the T47D human breast cancer cell line and decreases cell growth," Cancer Research, vol. 55, no. 23, pp. 5632-5636, 1995.

[11] R. Maneckjee, R. Biswas, and B. K. Vonderhaar, "Binding of opioids to human MCF-7 breast cancer cells and their effects on growth," Cancer Research, vol. 50, no. 8, pp. 2234-2238, 1990.

[12] M. Kampa, E. Bakogeorgou, A. Hatzoglou, A. Damianaki, P.-M. Martin, and E. Castanas, "Opioid alkaloids and casomorphin peptides decrease the proliferation of prostatic cancer cell lines (LNCaP, PC3 and DU145) through a partial interaction with opioid receptors," European Journal of Pharmacology, vol. 335, no. 2-3, pp. 255-265, 1997.

[13] I. Tegeder, S. Grösch, A. Schmidtko et al., "G protein-independent $\mathrm{G} 1$ cell cycle block and apoptosis with morphine in adenocarcinoma cells: involvement of p53 phosphorylation," Cancer Research, vol. 63, no. 8, pp. 1846-1852, 2003.

[14] M. P. Yeager and T. A. Colacchio, "Effect of morphine on growth of metastatic colon cancer in vivo," Archives of Surgery, vol. 126, no. 4, pp. 454-456, 1991.

[15] T. Sasamura, S. Nakamura, Y. Iida et al., "Morphine analgesia suppresses tumor growth and metastasis in a mouse model of cancer pain produced by orthotopic tumor inoculation," European Journal of Pharmacology, vol. 441, no. 3, pp. 185-191, 2002.

[16] Y. Harimaya, K. Koizumi, T. Andoh, H. Nojima, Y. Kuraishi, and I. Saiki, "Potential ability of morphine to inhibit the adhesion, invasion and metastasis of metastatic colon 26-L5 carcinoma cells," Cancer Letters, vol. 187, no. 1-2, pp. 121-127, 2002.

[17] R. H. Simon and T. E. Arbo, "Morphine increases metastatic tumor growth," Brain Research Bulletin, vol. 16, no. 3, pp. 363$367,1986$.

[18] J. W. Lewis, Y. Shavit, and G. W. Terman, "Apparent involvement of opioid peptides in stress-induced enhancement of tumor growth," Peptides, vol. 4, no. 5, pp. 635-638, 1983.

[19] M. G. Sergeeva, Z. V. Grishina, and S. D. Varfolomeyev, "Morphine effect on proliferation of normal and tumor cells of immune origin," Immunology Letters, vol. 36, no. 2, pp. 215-218, 1993.

[20] K. Gupta, S. Kshirsagar, L. Chang et al., "Morphine stimulates angiogenesis by activating proangiogenic and survival-promoting signaling and promotes breast tumor growth," Cancer Research, vol. 62, no. 15, pp. 4491-4498, 2002.

[21] M. Farooqui, Y. Li, T. Rogers et al., "COX-2 inhibitor celecoxib prevents chronic morphine-induced promotion of angiogenesis, tumour growth, metastasis and mortality, without compromising analgesia," British Journal of Cancer, vol. 97, no. 11, pp. 1523-1531, 2007.

[22] M. Ishikawa, K. Tanno, A. Kamo, Y. Takayanagi, and K.-I. Sasaki, "Enhancement of tumor growth by morphine and its possible mechanism in mice," Biological and Pharmaceutical Bulletin, vol. 16, no. 8, pp. 762-766, 1993.

[23] J. Zong and G. M. Pollack, "Morphine antinociception is enhanced in mdrla gene-deficient mice," Pharmaceutical Research, vol. 17, no. 6, pp. 749-753, 2000.

[24] M. Iglesias, M. F. Segura, J. X. Comella, and G. Olmos, “ $\mu$ opioid receptor activation prevents apoptosis following serum 
withdrawal in differentiated SH-SY5Y cells and cortical neurons via phosphatidylinositol 3-kinase," Neuropharmacology, vol. 44, no. 4, pp. 482-492, 2003.

[25] K. Gach, J. Szemraj, J. Fichna, M. Piestrzeniewicz, D. S. Delbro, and A. Janecka, "The influence of opioids on urokinase plasminogen activator on protein and mRNA level in MCF-7 breast cancer cell line," Chemical Biology and Drug Design, vol. 74, no. 4, pp. 390-396, 2009.

[26] P. A. Singleton and J. Moss, "Effect of perioperative opioids on cancer recurrence: a hypothesis," Future Oncology, vol. 6, no. 8, pp. 1237-1242, 2010.

[27] C. Chen, M. Farooqui, and K. Gupta, "Morphine stimulates vascular endothelial growth factor-like signaling in mouse retinal endothelial cells," Current Neurovascular Research, vol. 3, no. 3, pp. 171-180, 2006.

[28] D. R. Green and J. C. Reed, "Mitochondria and apoptosis," Science, vol. 281, no. 5381, pp. 1309-1312, 1998.

[29] A. Ashkenazi and V. M. Dixit, "Apoptosis control by death and decoy receptors," Current Opinion in Cell Biology, vol. 11, no. 2, pp. 255-260, 1999.

[30] D. Hanahan and R. A. Weinberg, "The hallmarks of cancer," Cell, vol. 100, no. 1, pp. 57-70, 2000.

[31] M. O. Hengartner, "The biochemistry of apoptosis," Nature, vol. 407, no. 6805, pp. 770-776, 2000.

[32] P.-N. Hsiao, M.-C. Chang, W.-F. Cheng et al., "Morphine induces apoptosis of human endothelial cells through nitric oxide and reactive oxygen species pathways," Toxicology, vol. 256, no. 1-2, pp. 83-91, 2009.

[33] A. A. Kapasi, S. A. Coscia, M. P. Pandya, and P. C. Singhal, "Morphine modulates HIV-1 gp160-induced murine macrophage and human monocyte apoptosis by disparate ways," Journal of Neuroimmunology, vol. 148, no. 1-2, pp. 86-96, 2004.

[34] X. Lin, Q. Li, Y.-J. Wang et al., "Morphine inhibits doxorubicininduced reactive oxygen species generation and nuclear factor $\kappa \mathrm{B}$ transcriptional activation in neuroblastoma SH-SY5Y cells," Biochemical Journal, vol. 406, no. 2, pp. 215-221, 2007.

[35] R. Arerangaiah, N. Chalasani, A. M. Udager et al., "Opioids induce renal abnormalities in tumor-bearing mice," NephronExperimental Nephrology, vol. 105, no. 3, pp. e80-e89, 2007.

[36] D. Salvemini, T. P. Misko, J. L. Masferrer, K. Seibert, M. G. Currie, and P. Needleman, "Nitric oxide activates cyclooxygenase enzymes," Proceedings of the National Academy of Sciences of the United States of America, vol. 90, no. 15, pp. 7240-7244, 1993.

[37] E. Nédélec, A. Abid, C. Cipolletta et al., "Stimulation of cyclooxygenase-2-activity by nitric oxide-derived species in rat chondrocyte: lack of contribution to loss of cartilage anabolism," Biochemical Pharmacology, vol. 61, no. 8, pp. 965-978, 2001.

[38] M. Farooqui, Z. H. Geng, E. J. Stephenson, N. Zaveri, D. Yee, and K. Gupta, "Naloxone acts as an antagonist of estrogen receptor activity in MCF-7 cells," Molecular Cancer Therapeutics, vol. 5, no. 3, pp. 611-620, 2006.

[39] D. Salvemini, K. Seibert, J. L. Masferrer, T. P. Misko, M. G. Currie, and P. Needleman, "Endogenous nitric oxide enhances prostaglandin production in a model of renal inflammation," Journal of Clinical Investigation, vol. 93, no. 5, pp. 1940-1947, 1994.

[40] R. J. Griffin, B. W. Williams, R. Wild, J. M. Cherrington, H. Park, and C. W. Song, "Simultaneous inhibition of the receptor kinase activity of vascular endothelial, fibroblast, and platelet-derived growth factors suppresses tumor growth and enhances tumor radiation response," Cancer Research, vol. 62, no. 6, pp. 17021706, 2002.
[41] K. M. Leahy, R. L. Ornberg, Y. Wang, B. S. Zweifel, A. T. Koki, and J. L. Masferrer, "Cyclooxygenase-2 inhibition by celecoxib reduces proliferation and induces apoptosis in angiogenic endothelial cells in vivo," Cancer Research, vol. 62, no. 3, pp. 625631, 2002.

[42] S. H. Chang, C. H. Liu, R. Conway et al., "Role of prostaglandin E2-dependent angiogenic switch in cyclooxygenase 2-induced breast cancer progression," Proceedings of the National Academy of Sciences of the United States of America, vol. 101, no. 2, pp. 591596, 2004.

[43] C.-F. Lam, Y.-C. Liu, F.-L. Tseng et al., "High-dose morphine impairs vascular endothelial function by increased production of superoxide anions," Anesthesiology, vol. 106, no. 3, pp. 532537, 2007.

[44] J. Blebea, J. E. Mazo, T. K. Kihara et al., "Opioid growth factor modulates angiogenesis," Journal of Vascular Surgery, vol. 32, no. 2, pp. 364-373, 2000.

[45] C.-F. Lam, P.-J. Chang, Y.-S. Huang et al., "Prolonged use of high-dose morphine impairs angiogenesis and mobilization of endothelial progenitor cells in mice," Anesthesia and Analgesia, vol. 107, no. 2, pp. 686-692, 2008.

[46] J. L. Martin, R. Charboneau, R. A. Barke, and S. Roy, "Chronic morphine treatment inhibits LPS-induced angiogenesis: implications in wound healing," Cellular Immunology, vol. 265, no. 2, pp. 139-145, 2010.

[47] S. Roy, S. Balasubramanian, J. Wang, Y. Chandrashekhar, R. Charboneau, and R. Barke, "Morphine inhibits VEGF expression in myocardial ischemia," Surgery, vol. 134, no. 2, pp. 336$344,2003$.

[48] L. Koodie, S. Ramakrishnan, and S. Roy, "Morphine suppresses tumor angiogenesis through a HIF-1 $\alpha /$ p38MAPK pathway," American Journal of Pathology, vol. 177, no. 2, pp. 984-997, 2010.

[49] N. Faramarzi, A. Abbasi, S. M. Tavangar, M. Mazouchi, and A. R. Dehpour, "Opioid receptor antagonist promotes angiogenesis in bile duct ligated rats," Journal of Gastroenterology and Hepatology, vol. 24, no. 7, pp. 1226-1229, 2009.

[50] R. L. Shapiro, J. G. Duquette, D. F. Roses et al., "Induction of primary cutaneous melanocytic neoplasms in urokinasetype plasminogen activator (uPA)-deficient and wild-type mice: cellular blue nevi invade but do not progress to malignant melanoma in uPA-deficient animals," Cancer Research, vol. 56, no. 15, pp. 3597-3604, 1996.

[51] J. A. Engbring and H. K. Kleinman, "The basement membrane matrix in malignancy," Journal of Pathology, vol. 200, no. 4, pp. 465-470, 2003.

[52] M. S. Widel and M. Widel, "Mechanisms of metastasis and molecular markers of malignant tumor progression. I. Colorectal cancer," Postępy Higieny i Medycyny Doświadczalnej, vol. 60, pp. 453-470, 2006.

[53] C. C. Lynch and L. M. Matrisian, "Matrix metalloproteinases in tumor-host cell communication," Differentiation, vol. 70, no. 910, pp. 561-573, 2002.

[54] K. Gach, J. Szemraj, A. Wyrębska, and A. Janecka, “The influence of opioids on matrix metalloproteinase-2 and -9 secretion and mRNA levels in MCF-7 breast cancer cell line," Molecular Biology Reports, vol. 38, no. 2, pp. 1231-1236, 2011.

[55] G. Nylund, A. Pettersson, C. Bengtsson, A. Khorram-Manesh, S. Nordgren, and D. S. Delbro, "Functional expression of $\mu$-opioid receptors in the human colon cancer cell line, HT-29, and their localization in human colon," Digestive Diseases and Sciences, vol. 53, no. 2, pp. 461-466, 2008. 


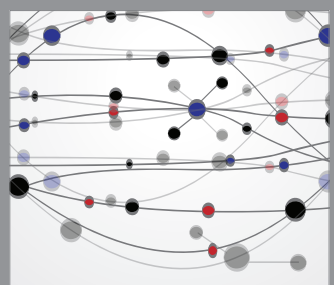

The Scientific World Journal
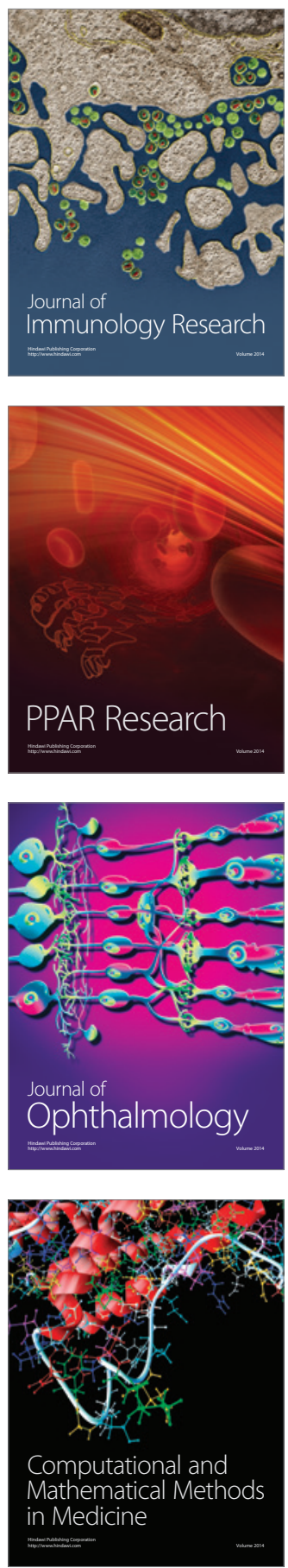

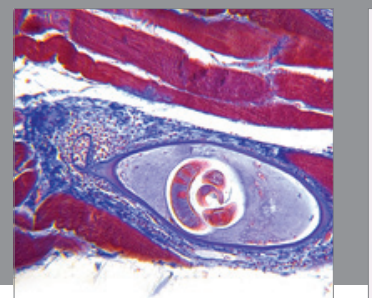

Gastroenterology

Research and Practice
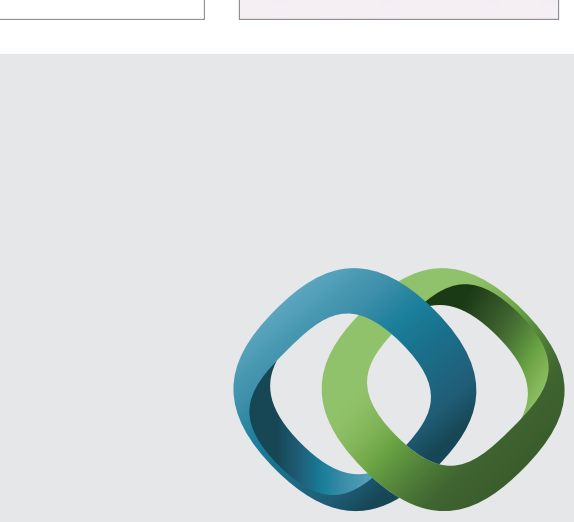

\section{Hindawi}

Submit your manuscripts at

http://www.hindawi.com
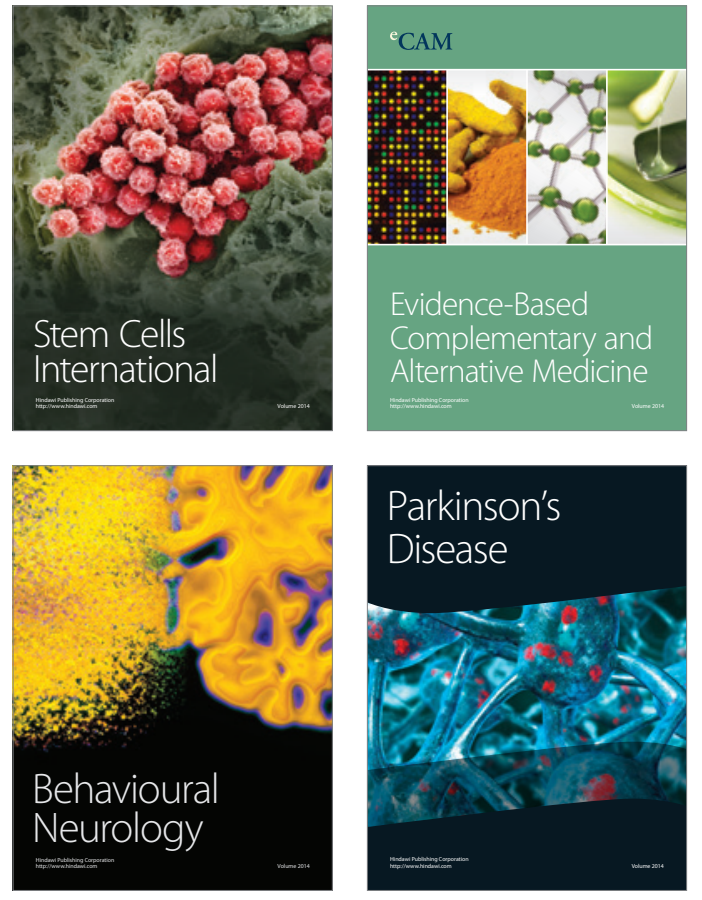
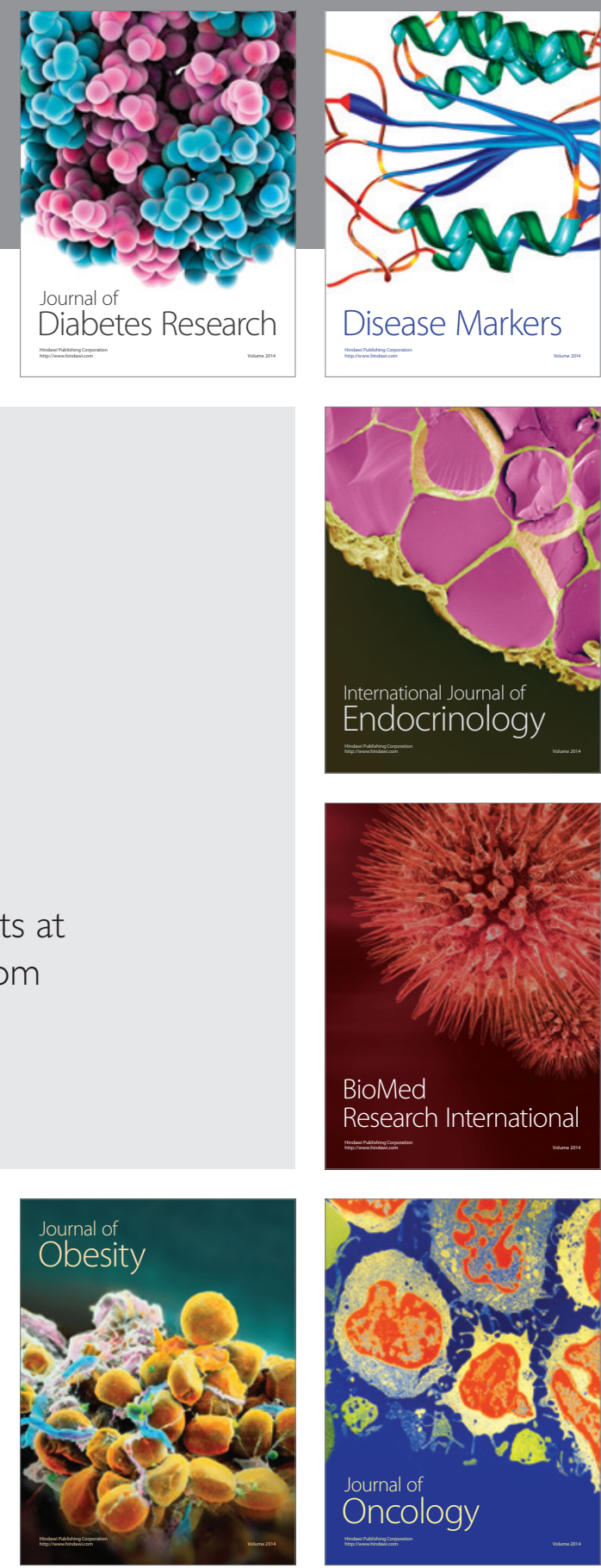

Disease Markers
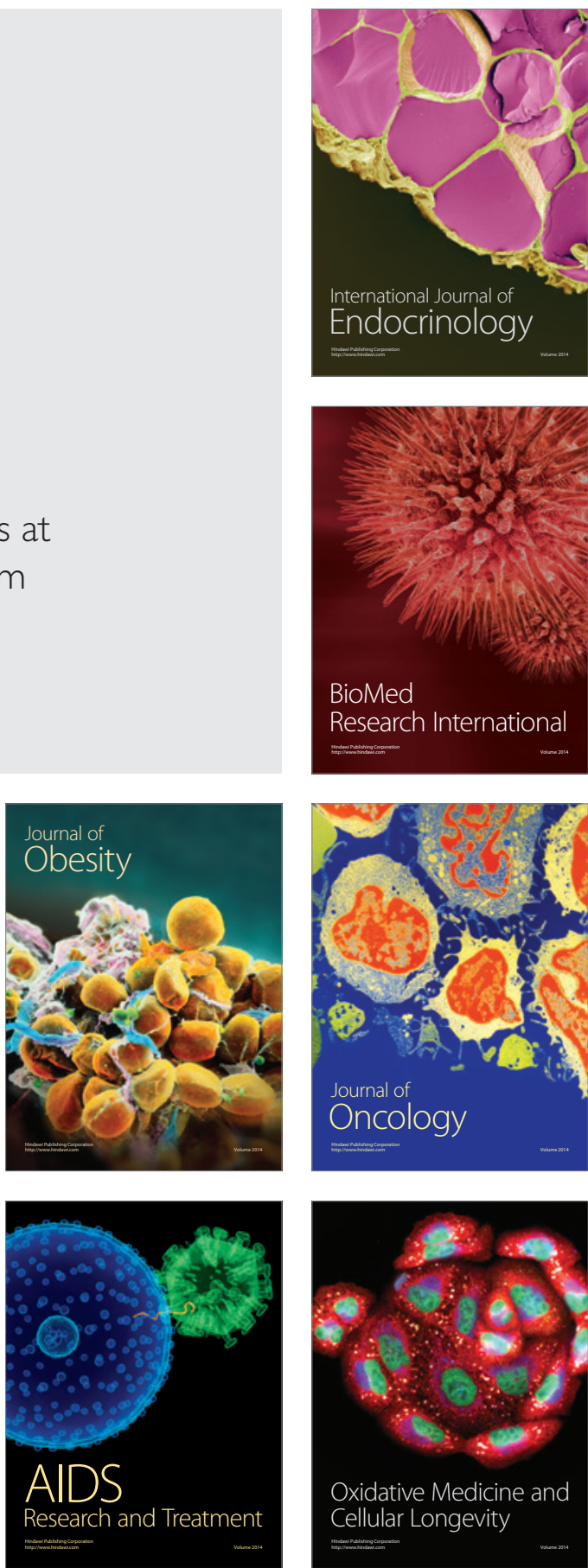